\title{
In vivo staging of regional amyloid progression in healthy middle-aged to older people at risk of Alzheimer's disease
}

Fedor Levin' ${ }^{1}$, Irina Jelistratova' ${ }^{1}$, Tobey J. Betthauser ${ }^{2,3}$, Ozioma Okonkwo ${ }^{2,3}$, Sterling C. Johnson 2,3,4,5, Stefan J. Teipel ${ }^{1,6}$ and Michel J. Grothe ${ }^{1,7^{*}}$ (e)

\begin{abstract}
Background: We investigated regional amyloid staging characteristics in ${ }^{11} \mathrm{C}-\mathrm{PiB}$-PET data from middle-aged to older participants at elevated risk for AD enrolled in the Wisconsin Registry for Alzheimer's Prevention.

Methods: We analyzed partial volume effect-corrected ${ }^{11} \mathrm{C}$-PiB-PET distribution volume ratio maps from 220 participants (mean age $=61.4$ years, range 46.9-76.8 years). Regional amyloid positivity was established using region-specific thresholds. We used four stages from the frequency-based staging of amyloid positivity to characterize individual amyloid deposition. Longitudinal PET data was used to assess the temporal progression of stages and to evaluate the emergence of regional amyloid positivity in participants who were amyloid-negative at baseline. We also assessed the effect of amyloid stage on longitudinal cognitive trajectories.
\end{abstract}

Results: The staging model suggested progressive accumulation of amyloid from associative to primary neocortex and gradually involving subcortical regions. Longitudinal PET measurements supported the cross-sectionally estimated amyloid progression. In mixed-effects longitudinal analysis of cognitive follow-up data obtained over an average period of 6.5 years following the baseline PET measurement, amyloid stage II showed a faster decline in executive function, and advanced amyloid stages (III and IV) showed a faster decline across multiple cognitive domains compared to stage 0 .

Conclusions: Overall, the ${ }^{11} \mathrm{C}$-PiB-PET-based staging model was generally consistent with previously derived models from ${ }^{18}$ F-labeled amyloid PET scans and a longitudinal course of amyloid accumulation. Differences in longitudinal cognitive decline support the potential clinical utility of in vivo amyloid staging for risk stratification of the preclinical phase of $A D$ even in middle-aged to older individuals at risk for $A D$.

Keywords: Amyloid- $\beta$, Amyloid PET, ${ }^{11} \mathrm{C}-\mathrm{PiB}$, Amyloid staging

*Correspondence: mgrothe@us.es

${ }^{7}$ Unidad de Trastornos del Movimiento, Servicio de Neurología y Neurofisiología Clínica, Instituto de Biomedicina de Sevilla, Hospital Universitario Virgen del Rocío/CSIC/Universidad de Sevilla, s/n, 41013 Seville, Spain

Full list of author information is available at the end of the article

\section{Introduction}

Aggregates of amyloid- $\beta(A \beta)$ protein are an important early histopathological hallmark of Alzheimer's disease (AD). Previous research has demonstrated that early amyloid accumulation can be observed in cognitively healthy people long before the onset of dementia [1-4]. Deposition of amyloid in the brain can be measured in vivo using positron emission tomography (PET) with amyloid-sensitive radiotracers such as ${ }^{11} \mathrm{C}$ Pittsburgh 
Compound $\mathrm{B}(\mathrm{PiB})$ or 2 nd-generation ${ }^{18} \mathrm{~F}$-labeled radiotracers. These tracers show high sensitivity and specificity when compared to the neuropathological gold standard [5-8]. However, in contrast to the established neuropathological staging schemes of regionally progressing amyloid pathology [4, 9], clinical PET-based in vivo assessment of amyloid pathology is most commonly limited to a binary classification into positive or negative categories based on the global amyloid PET signal.

In a previous study, we developed a data-driven in vivo staging model of regional amyloid progression which was based on the frequency of regional amyloid positivity in ${ }^{18} \mathrm{~F}$-florbetapir PET scans of cognitively unimpaired older adults [10]. The staging model was also validated in an independent cohort of participants with subjective cognitive decline [11]. In both studies, amyloid staging identified early stages of amyloid accumulation that were not detected by the conventional binary classification approach based on global PET signal. In an imaging-toautopsy correlation study, the staging was also associated with neuropathologically defined phases of amyloid deposition [12]. Moreover, a stage-proportional risk for clinical disease progression could be demonstrated across cohorts [13]. Taken together, these findings indicate the potential usefulness of in vivo amyloid staging for a pathologic stratification of preclinical AD.

However, it is clear that the nuances of the methodological approach, such as radiotracer selection, definition of amyloid positivity cutoffs, choice of reference region, and the use of partial volume effects (PVE) correction, can affect the quantitative amyloid PET imaging results and the regional staging outcomes $[8,14,15]$. Analysis of amyloid-PET scans of young healthy adults who are highly unlikely to exhibit cerebral amyloid deposition demonstrated a considerable variation in regional uptake values [16]. This indicates regionally varying noise levels in amyloid-PET signals and argues for the use of regionspecific amyloid positivity cutoffs in the staging model [17]. It was previously reported that global cortical signals of ${ }^{11} \mathrm{C}$-PiB-PET and ${ }^{18} \mathrm{~F}$-based amyloid-PET imaging data obtained from the same individuals were highly correlated [18]. However, subtle differences in binding affinity to amyloid and non-specific white matter binding between different tracers have also been demonstrated [19-22], which could potentially affect their ability to detect early diffuse and later neuritic types of $A \beta$ protein aggregates and could thus also result in differing regional patterns of early-stage amyloid pathology. Finally, the characteristics of the studied cohort could likely affect the outcome of the analysis. Particularly, age is expected to influence the ability of amyloid staging to predict cognitive decline.
In the present study, we determined the regional amyloid staging characteristics in dynamically acquired ${ }^{11} \mathrm{C}$-PiB-PET data from middle-aged to older individuals enrolled in the Wisconsin Registry for Alzheimer's Prevention (WRAP) study who are at increased risk for $\mathrm{AD}$ due to a family history of AD dementia. Of note, this cohort was considerably younger than the cohorts investigated previously. We further assessed the longitudinal validity of the cross-sectionally estimated staging model by analyzing individual stage transitions in serial PET scans and additionally analyzed the first sites of longitudinal amyloid accumulation in participants without any evidence of regional amyloid positivity at baseline. Finally, we assessed whether cross-sectionally estimated amyloid stages were predictive of longitudinal cognitive trajectories in this relatively young at-risk cohort.

\section{Materials and methods \\ Participants}

We studied 220 participants (mean age $=61.4$ years, range 46.9-76.8 years, 151 females) selected from the WRAP cohort based on the availability of at least one ${ }^{11} \mathrm{C}$-PiB-PET scan. Participants underwent the first ${ }^{11} \mathrm{C}$-PiB-PET scan on average 6.4 years after the first neuropsychological assessment. Among participants, 159 (72\%) had at least one biological parent diagnosed with $\mathrm{AD}$ dementia. A subset of 157 participants had available imaging data from a second ${ }^{11} \mathrm{C}$-PiB-PET scan (average time interval of 2.5 years since the first ${ }^{11} \mathrm{C}$-PiB-PET scan), and 60 had additional imaging data from a third ${ }^{11} \mathrm{C}$-PiB-PET scan (average time interval of 6.1 years since the first scan). Participants were healthy and unimpaired at baseline. Eight participants had a diagnosis of clinical $\mathrm{MCI}$ at a WRAP study visit prior to the first ${ }^{11} \mathrm{C}-\mathrm{PiB}-\mathrm{PET}$ scan, and one had a dementia diagnosis shortly after the first ${ }^{11} \mathrm{C}$-PiB-PET scan. We excluded the data from these participants from the longitudinal analysis of cognitive performance. Out of 189 participants with available $A P O E-\varepsilon 4$ data, $34 \%$ were $A P O E-\varepsilon 4$ positive.

\section{Imaging data}

Acquisition of ${ }^{11} \mathrm{C}-\mathrm{PiB}-\mathrm{PET}$ and MRI imaging data in the WRAP cohort has been described in detail previously [23]. Briefly, ${ }^{11} \mathrm{C}-\mathrm{PiB}-\mathrm{PET}$ scans were acquired in a 3-D mode with a dynamic 70-min acquisition protocol after an injection of a $15-\mathrm{mCi}$ target dose of ${ }^{11} \mathrm{C}-\mathrm{PiB}$ bolus. Dynamic acquisition frames consisted of 17 time frames, including $5 \times 2 \mathrm{~min}$ and $12 \times 5$ min frames. A filtered back-projection algorithm was used for reconstructing the data. For anatomical reference, a highresolution T1-weighted MRI scan was acquired using a 3.0-Tesla GE MR750 scanner with an 8 or 32 channel head coil. The 3-D inversion recovery prepared fast 
spoiled gradient-echo sequence had the following parameters: inversion time $(\mathrm{TI})=450 \mathrm{~ms}$, repetition acquisition matrix $=256 \times 256 \times 156 \mathrm{~mm}$, field of view (FOV) $=256 \mathrm{~mm}$, and slice thickness $=1.0 \mathrm{~mm}$. The reconstructed time series of ${ }^{11} \mathrm{C}$-PiB-PET data were realigned, corrected for motion, de-noised, and coregistered to the subject's T1-weighted MRI scan based on co-registration of the time-integrated PET scan utilizing the Statistical Parametric Mapping software (SPM12; www.fil.ion.ucl. ac.uk/spm). Parametric distribution volume ratio (DVR) maps were generated using Logan graphical analysis methods [24, 25] with $t^{*}=35 \mathrm{~min}$ and cerebellar gray matter as a reference region of non-displaceable binding.

\section{Image analysis}

The imaging data were further pre-processed for regional staging analysis using previously described procedures [10]. MRI images were segmented into different tissue types and spatially normalized to a customized aging/ AD-specific reference template space [26] using the highdimensional spatial registration algorithm DARTEL [27]. ${ }^{11} \mathrm{C}-\mathrm{PiB}-\mathrm{PET}$ DVR maps were corrected for PVE using the 3-compartment "Müller-Gärtner" method in the subject's native space [28, 29], and then spatially normalized to the reference template space using transformation parameters from the corresponding MRI. Regional DVR values were then extracted from 52 regions of interest within the reference template defined using the HarvardOxford atlas, which included 48 cortical regions, as well as the hippocampus, amygdala, striatum, and thalamus. We also extracted the average global non-PVE-corrected and PVE-corrected ${ }^{11} \mathrm{C}$-PiB-PET DVR signal within a cortical composite mask [30].

We used a two-dimensional Gaussian mixture model (GMM) approach utilizing regional and global mean PVE-corrected DVR values to establish region-specific thresholds for amyloid positivity. Analogous to previous studies using one-dimensional GMM [8,31, 32], we fit low and high amyloid distributions for each region. The two-dimensional GMM approach is different in that it estimates the distribution of two variables at once so that the contribution of each regional DVR value to the low or high amyloid distribution is estimated in conjunction with the global amyloid signal of each participant. This approach was intended to decrease the susceptibility of the procedure to the potential noisiness of regional signal resulting in more robust and biologically plausible regional estimates. Regional thresholds were defined as 1.65 standard deviations above the mean value of the low A $\beta$ distribution corresponding to the 95 th percentile $[16$, 33].

In analogy to neuropathological staging models and our previous PET-based staging study, we determined a regional amyloid progression model based on the frequency of regional amyloid positivity across individuals as an indicator of progressive temporal involvement $[4,9$, 10, 34]. Regional frequencies of amyloid positivity were calculated from the baseline PET data using 10,000 bootstrap resamples, and the obtained range of frequencies was split into four equal parts to obtain a discrete stage model of amyloid progression across four larger anatomical divisions [10, 17].

In sensitivity analyses, we additionally assessed the effect of alternative PET processing methods as well as strategies for estimating regional positivity thresholds. Among PET processing strategies, we assessed the effects of (i) using more commonly obtained standard uptake value ratio (SUVR) images $[8,21]$ instead of the DVR images obtained from the dynamic PET acquisitions, (ii) using non-PVE-corrected PET data, and (iii) using a constant vs region-specific thresholds. Alternative strategies for estimating regional positivity thresholds included (i) a 1-dimensional GMM approach based on regional values only and (ii) a regional resampling approach in a subsample of the 20 youngest, $A P O E$ - $\varepsilon 4$-negative subjects without familial history of $\mathrm{AD}$ (mean age $=59.8$ years, 16 females). For both of these methods, the thresholds were analogously estimated as 1.65 standard deviations above the mean value, and regional frequencies of amyloid positivity were calculated using 10,000 bootstrap resamples. The correspondence between the regional amyloid positivity frequencies derived from the different PET processing and cutoff derivation methods was assessed using pair-wise Spearman rank correlations.

Individual amyloid deposition profiles were staged according to the regional hierarchy indicated by the estimated amyloid progression model. For that, each of the four larger anatomical divisions defined by the 4-stage model was considered amyloid-positive if at least half of the included regions displayed a suprathreshold signal $[10,17]$. The individual stage was then determined based on the corresponding amyloid-positive anatomical divisions. For example, a classification of stage III requires positivity in anatomical divisions 1, 2, and 3, but not 4 . Participants whose regional amyloid positivity profile did not adhere to the expected regional hierarchy (e.g., positivity in anatomical division 2, but not in 1) were classified as non-stageable. For comparison, we dichotomized the ${ }^{11} \mathrm{C}$-PiB-PET scans into standard amyloid-positive/ negative categories based on a previously established threshold of 1.08 applied to the global composite DVR value in non-PVE-corrected data [8].

\section{Longitudinal imaging analysis}

The longitudinal validity of the cross-sectionally estimated regional amyloid staging model was assessed in 
two complementary analyses. First, we assessed individual longitudinal changes in amyloid stages from baseline to the furthest available follow-up PET scan. Among stageable participants at baseline, 155 had a follow-up ${ }^{11} \mathrm{C}$-PiB-PET scan with an average time delay of 4 years (range 1.7-7.7). In a complementary analysis independent from the estimated staging model, we assessed the first longitudinal appearance of regional amyloid positivity in subjects who had no suprathreshold signal in any of the 52 brain regions at baseline $(n=$ 64) by recording the regional amyloid positivity occurring at the follow-up ${ }^{11} \mathrm{C}$-PiB-PET scans, on average 3.9 years later (range $1.8-7.6$ years).

\section{Neuropsychological testing}

Finally, to examine the potential clinical relevance of the amyloid staging approach, we analyzed the longitudinal cognitive trajectories of participants at different amyloid stages using previously developed domain-specific and global cognitive composite scores [35]. These scores included a delayed recall composite (THEO-DEL-REC), an executive function composite (THEO-EXEC-FN), an immediate learning composite (THEO-IMM-LRN), and a global cognitive composite score-a three test version of the preclinical Alzheimer's cognitive composite (PACC3).

In order to assess the differences in future cognitive trajectories across in vivo amyloid stages, in the regression analysis, we selected neuropsychological scores obtained at visits taking place at the earliest 3 months before the first ${ }^{11} \mathrm{C}$-PiB-PET measurement and later. Five participants did not have available neuropsychological test scores after that time point and were excluded. The composite scores were only available from WRAP study visit 2 and onwards, because the more extensive cognitive testing required for the composite score calculation was not yet introduced at the first WRAP study visits. As a result, the closest WRAP visit with neuropsychological testing was on average 1.1 years after the first ${ }^{11} \mathrm{C}$-PiB-PET scan. We included cognitive data from a median of 3 WRAP study visits per participant conducted on average at 2.5-year intervals. The mean duration of the total follow-up was 6.5 years from the first ${ }^{11} \mathrm{C}$-PiB-PET scan until the last available cognitive assessment, with a maximum of 8.7 years of follow-up. Longitudinal trajectories of the four cognitive composite scores were analyzed using linear mixed-effects regression models implemented in R 3.6.0 [36]. The effect of the amyloid stage on longitudinal cognitive decline was assessed by the time $x$ amyloid stage interaction, controlled for age at the first analyzed WRAP visit, sex, and years of education.

\section{Results}

\section{Frequency-based staging model of regional amyloid progression}

Overall, 133 out of the 220 participants (60\%) demonstrated amyloid positivity in at least one brain region. According to the cross-sectional amyloid progression model based on regional frequencies (Fig. 1), amyloid deposition begins in the anterior and posterior cortical midline structures, the inferior temporal lobe, and lateral temporo-parietal association areas (stage I); stage II involves more extensive parts of the association cortex, particularly the lateral frontal areas, as well as the striatum; stage III is characterized by involvement of primary sensory-motor areas (pre- and postcentral gyrus), as well as of the medial temporal lobe (including hippocampus and amygdala) and temporal pole; and stage IV finally includes the occipital pole, remaining parts of the medial temporal lobe, and the thalamus.

We observed moderate to strong positive correlations between regional amyloid positivity frequencies estimated with three different methods for defining regional amyloid positivity thresholds (see Supplementary table 1 and Supplementary figure 1). In general, the two-dimensional and one-dimensional GMM approaches yielded highly correlated regional progression models (rho $=$ 0.78 ), whereas larger differences were observed when thresholds were derived from the 20 youngest participants (Spearman rank correlations rho $=0.63$ and 0.57, compared to 2D-GMM and 1D-GMM, respectively). Some of the most salient differences corresponded to a relatively earlier involvement (i.e., higher relative amyloid positivity frequencies) of some temporal lobe (Heschl's gyrus and planum temporale) and subcortical regions (thalamus, hippocampus, and amygdala; see Supplementary figure 1 for brain renderings of the respective amyloid positivity frequencies). We observed a moderate correlation between the current ${ }^{11} \mathrm{C}$-PiB-PET-based model and the previous regional frequency-based model derived from ${ }^{18} \mathrm{~F}$-Florbetapir-PET data in older cognitively normal participants from the ADNI cohort [10] (rho $=0.54, p<0.001)$. However, notable differences between the models were also evident, particularly, with respect to a relatively earlier involvement of medial parietal (stage I) and striatal regions (stage II) in the current ${ }^{11} \mathrm{C}$-PiB-PET-based model.

The results of the individual staging analysis and sample characteristics by stage are presented in Table 1. Thirtyseven participants $(17 \%)$ were amyloid-positive in any anatomical division, and four of these participants were non-stageable. As expected, higher amyloid stages corresponded to higher mean global DVR values, and 1.6\% of stage 0 participants, $36.7 \%$ of stage I participants, and all of stage II, stage III, and stage IV participants 


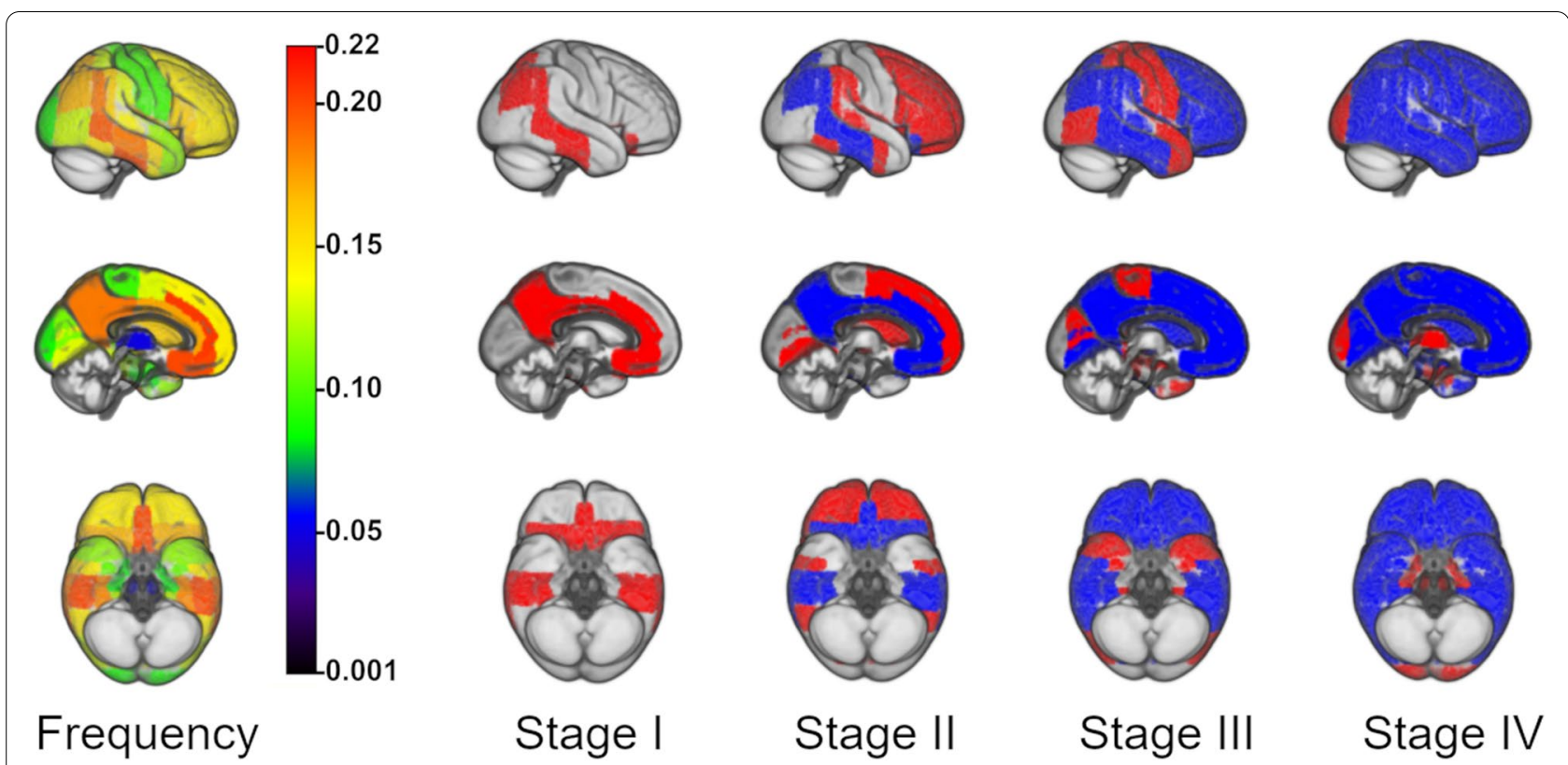

Fig. 1 Regional amyloid progression model and the derived staging scheme with 4 stages. Brain renderings on the left illustrate the frequency of regional amyloid positivity (color scale) from black/blue (lowest) to yellow/red (highest), which was used as an indicator of temporal progression. In the resulting staging scheme on the right, incremental stages (I-IV) are defined by an involvement of higher numbered anatomic divisions (in red) in addition to the affected areas of the previous stage (blue)

Table 1 Sample characteristics by regional amyloid stage

\begin{tabular}{llllllll}
\hline & Full sample & Stage $\mathbf{0}$ & Stage I & Stage II & Stage III & Stage IV & Non-stageable \\
\hline$n$ & 220 & 183 & 8 & 7 & 10 & 8 & 4 \\
Age, years (SD) & $61.4(6.2)$ & $60.7(6.2)$ & $67.5(6.0)$ & $64.5(3.5)$ & $63.7(2.8)$ & $65.2(4.8)$ & $64.7(8.9)$ \\
Sex, \% female & $69 \%$ & $68 \%$ & $75 \%$ & $57 \%$ & $80 \%$ & $75 \%$ & $50 \%$ \\
Education, years (SD) & $16.4(2.6)$ & $16.4(2.7)$ & $16.8(1.8)$ & $17.6(2.0)$ & $16.9(2.6)$ & $15.9(2.3)$ & $15.3(2.2)$ \\
APOE \&4 (\%) & $34 \%$ & $29 \%$ & $40 \%$ & $83 \%$ & $60 \%$ & $71 \%$ & $0 \%$ \\
Parental history of AD (\%) & $73 \%$ & $71 \%$ & $43 \%$ & $86 \%$ & $90 \%$ & $100 \%$ & $75 \%$ \\
Mean global ${ }^{11}$ C-PiB DVR & 1.03 & 0.98 & 1.08 & 1.19 & 1.32 & 1.46 & 1.07 \\
Global ${ }^{11}$ C-PiB DVR > 1.08 & $33(15 \%)$ & $3(1.64 \%)$ & $3(37.5 \%)$ & $7(100 \%)$ & $10(100 \%)$ & $8(100 \%)$ & $2(50 \%)$ \\
MMSE & $29.3(1.1)$ & $29.3(1.1)$ & $29.7(0.5)$ & $29.4(0.8)$ & $29.4(0.8)$ & $28.5(2.7)$ & $29.8(0.5)$ \\
Delayed recall composite score & $0.04(0.85)$ & $0.07(0.81)$ & $-0.02(0.83)$ & $0.23(0.92)$ & $-0.27(0.84)$ & $-0.59(1.73)$ & $0.09(0.96)$ \\
Executive function composite score & $-0.06(0.71)$ & $-0.01(0.69)$ & $0.22(0.25)$ & $-0.43(1.30)$ & $-0.60(0.56)$ & $-0.63(0.54)$ & $-0.06(0.52)$ \\
Immediate learning composite score & $0.04(0.82)$ & $0.06(0.80)$ & $0.11(0.71)$ & $0.21(0.89)$ & $-0.27(0.96)$ & $-0.27(1.09)$ & $0.25(1.11)$ \\
PACC3 composite score & $-0.03(0.78)$ & $0.00(0.78)$ & $0.29(0.55)$ & $0.07(0.69)$ & $-0.59(0.79)$ & $-0.43(0.93)$ & $-0.05(0.86)$ \\
\hline
\end{tabular}

Values for age, years of education, MMSE and cognitive composite scores are presented as means with standard deviation in parentheses. Please note that individuals with missing values were excluded from this summary

were classified as amyloid-positive based on the standard global amyloid signal threshold (Table 1). Two of the four non-stageable participants were also classified as amyloid-positive.

\section{Longitudinal analyses of regional amyloid progression}

Comparison of the stages determined at baseline and follow-up PET scans suggested that participants in each stage either remained stable at the baseline stage (81.3\% of 155 stageable participants with a follow-up scan, see Fig. 2) or showed a model-conform transition to a higher stage (15.5\%). One participant demonstrated a regression from stage IV at baseline to stage III at follow-up, and four participants (2.6\%) showed a progression pattern that violated the estimated regional hierarchy, i.e., classified as non-stageable at 


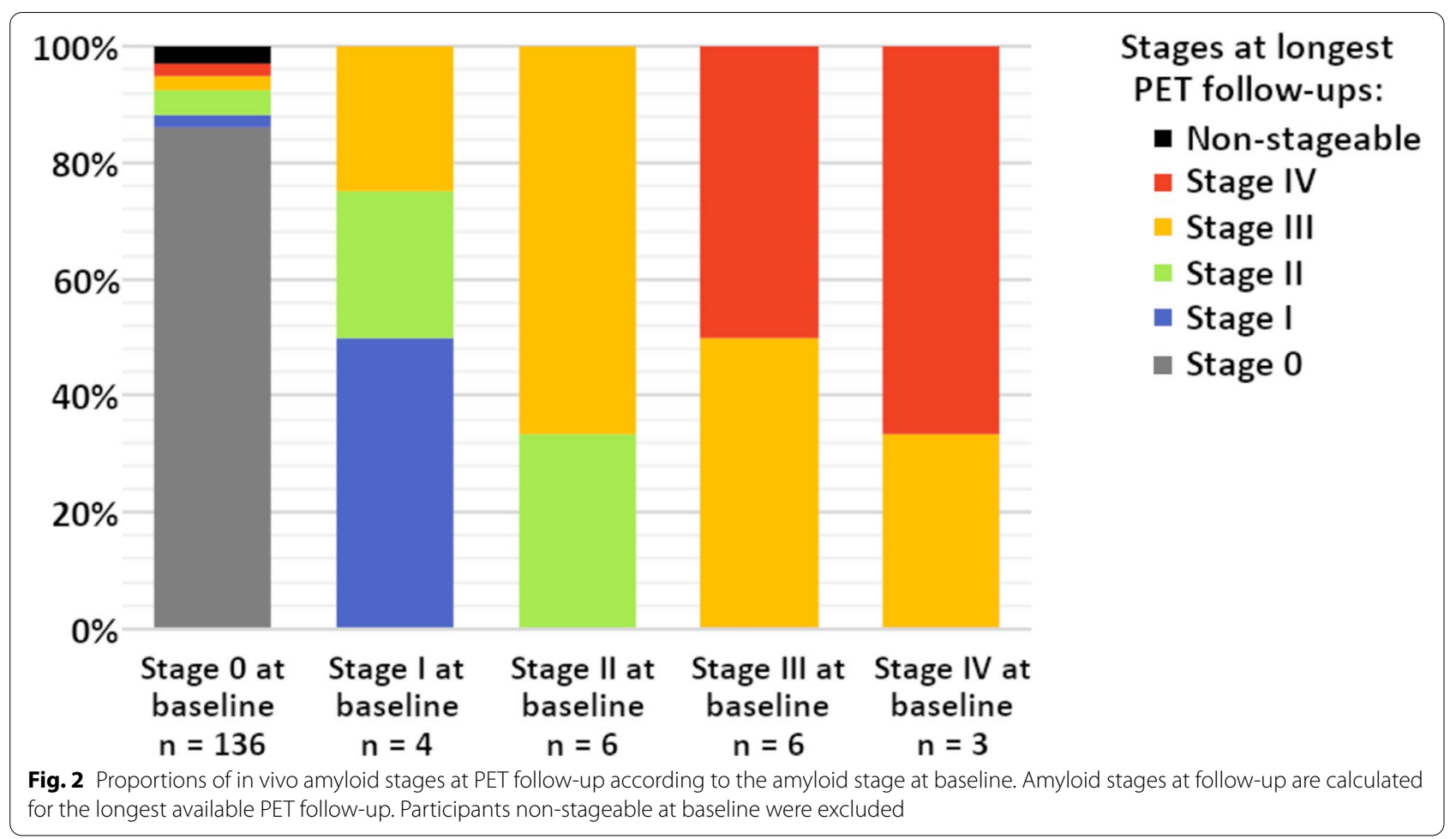

follow-up (all stage 0 at baseline). Characteristics of the subsample of participants who progressed to a higher stage compared to those who did not are presented in Supplementary table 2.

In the longitudinal analysis of participants who had no amyloid-positive regions at baseline and had follow-up scans $(n=64), 35$ participants $(55 \%)$ developed amyloid positivity in one or more regions over the follow-up period. Regional emergence of amyloid positivity was mostly localized to the inferior temporal (18.8\%) and temporal fusiform gyrus (12.5\%), the anterior parahippocampal gyrus (10.9\%), and the posterior cingulate cortex $(10.9 \%)$ but was also observed in the medial frontal and lateral temporal areas in smaller subsets of individuals (see Fig. 3).

\section{Effect of amyloid stage on cognitive decline}

Results from the mixed-effects regression models indicated differential trajectories of cognitive decline depending on the baseline amyloid stage (Fig. 4, Table 2). Compared to stage 0, stages II and III showed a faster decline in executive function, whereas stages III and IV showed a faster decline in immediate learning, delayed recall, and global cognition as measured by the PACC3 score.

\section{Discussion}

In the current study, we established a regional staging model of progressive amyloid accumulation in cross-sectional ${ }^{11} \mathrm{C}$-PiB-PET data from a sample of middle-aged to older individuals at elevated risk for $\mathrm{AD}$, assessed its longitudinal validity in serial PET scans, and examined its predictive value for forecasting longitudinal cognitive decline. The estimated amyloid staging model suggested a regional hierarchy where amyloid deposition begins in anterior and posterior cortical midline structures, lateral temporo-parietal association areas, and the inferior temporal lobe (stage I), and then sequentially affects the remaining association cortex, the striatum, primary sensory-motor areas, and finally the medial temporal lobe and thalamus (Fig. 1). Individual stage transitions in longitudinal PET data largely adhered to this crosssectionally estimated staging model (Fig. 2), and complementary analysis of longitudinal amyloid accumulation in individuals without any evidence of regional amyloid deposition at baseline corroborated an early affection of the estimated stage I regions, particularly of the inferior temporal lobe and the posterior cingulate. An early increase of amyloid signal in temporal lobe regions has been reported in several previous studies using ${ }^{18} \mathrm{~F}$-based radiotracers [37-39] and was also a consistent feature in our previously developed regional amyloid staging 


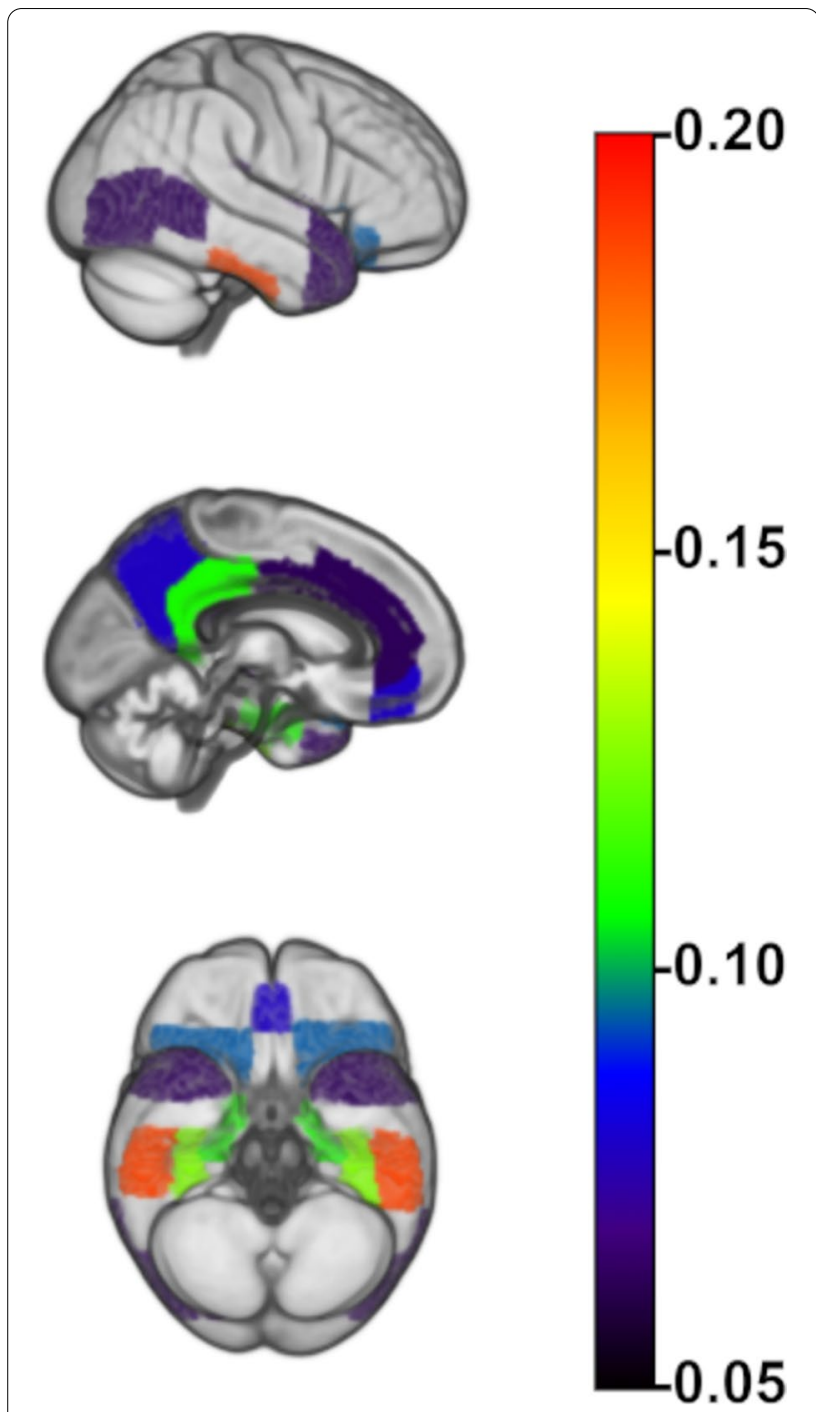

Fig. 3 The first longitudinal appearance of regional amyloid positivity. Color scale reflects the probability of a brain region to become amyloid-positive over the longest available PET follow-up in participants who were completely amyloid-negative at baseline. Only regions with probabilities higher than 0.05 are shown

models based on ${ }^{18} \mathrm{~F}$-florbetapir PET data from the ADNI cohort $[10,17]$. While this is also consistent with early neuropathological estimates of regional amyloid progression [9], other amyloid-PET studies have more consistently pointed to the anterior and posterior cingulate as the earliest amyloid accumulating regions in $\mathrm{AD}[8,40$, 41]. Regarding this discrepancy, it was hypothesized that the early amyloid-PET signal increases in the temporal lobe may reflect some sort of "physiological" age-related amyloid deposition, whereas amyloid accumulation in anterior and posterior midline structures is more closely associated with progressive AD pathology [17, 39].
The overall progression pattern from cortical association areas over primary sensory-motor areas to the medial temporal lobe and subcortical structures is largely consistent with our previously estimated staging models for ${ }^{18} \mathrm{~F}$-florbetapir PET. One notable difference of the current results is the relatively early affection of the striatum (stage II), preceding affection of primary sensory-motor areas and the medial temporal lobe (stage III). Early amyloid deposition in the striatum has been reported in PiB-PET data from autosomal-dominant variants of AD [42-44]. In contrast, striatal amyloid deposits are estimated to correspond to relatively advanced stages of amyloid pathology in sporadic AD (Thal phase 3) [4]. One previous PiB-PET staging study found elevated striatal PiB-PET signal only among individuals who already had elevated signal in neocortical association areas, but primary sensory-motor cortical areas or the medial temporal lobe were not assessed in that study [45]. It remains to be determined whether the observed difference in striatal involvement relates to actual differences in regional radiotracer binding characteristics or rather reflects specifics of the different cohorts used for estimating the staging models.

Similar to our previous staging studies $[10,11,17]$, the current PiB-PET findings suggest that the estimated amyloid staging model provides a higher sensitivity for early amyloid detection compared to a more conventional binary classification of subjects into amyloid-negative or amyloid-positive categories. Only $37.5 \%$ of the stage I participants were categorized as amyloid-positive by a standard global amyloid threshold proposed for binarization of the ${ }^{11} \mathrm{C}-\mathrm{PiB}-\mathrm{PET}$ DVR data [8].

A major strength of our current study is that we were able to study the effect of the estimated amyloid stages on long-term cognitive trajectories assessed over an average of 6.5 years of clinical follow-up. Here, we could demonstrate that risk of cognitive decline was proportional to the estimated amyloid stage. While individuals with advanced amyloid stages III and IV at baseline showed a faster decline in both the PACC3 global composite and domain-specific scores, even the earlier stage II showed a significantly faster decline in executive function compared to individuals without evidence of regional amyloid pathology (stage 0). The somewhat counterintuitive finding that stage IV participants did not also demonstrate a significantly faster decline in executive function may possibly be due to the relatively low numbers of participants categorized into this stage and the high variability in domain-specific cognitive decline in the preclinical phase of $\mathrm{AD}$ [46]. In contrast to our current findings, some previous studies reported a relatively memory-specific cognitive decline in function of higher global amyloid-PET levels among middle-aged and older adults [47]. 


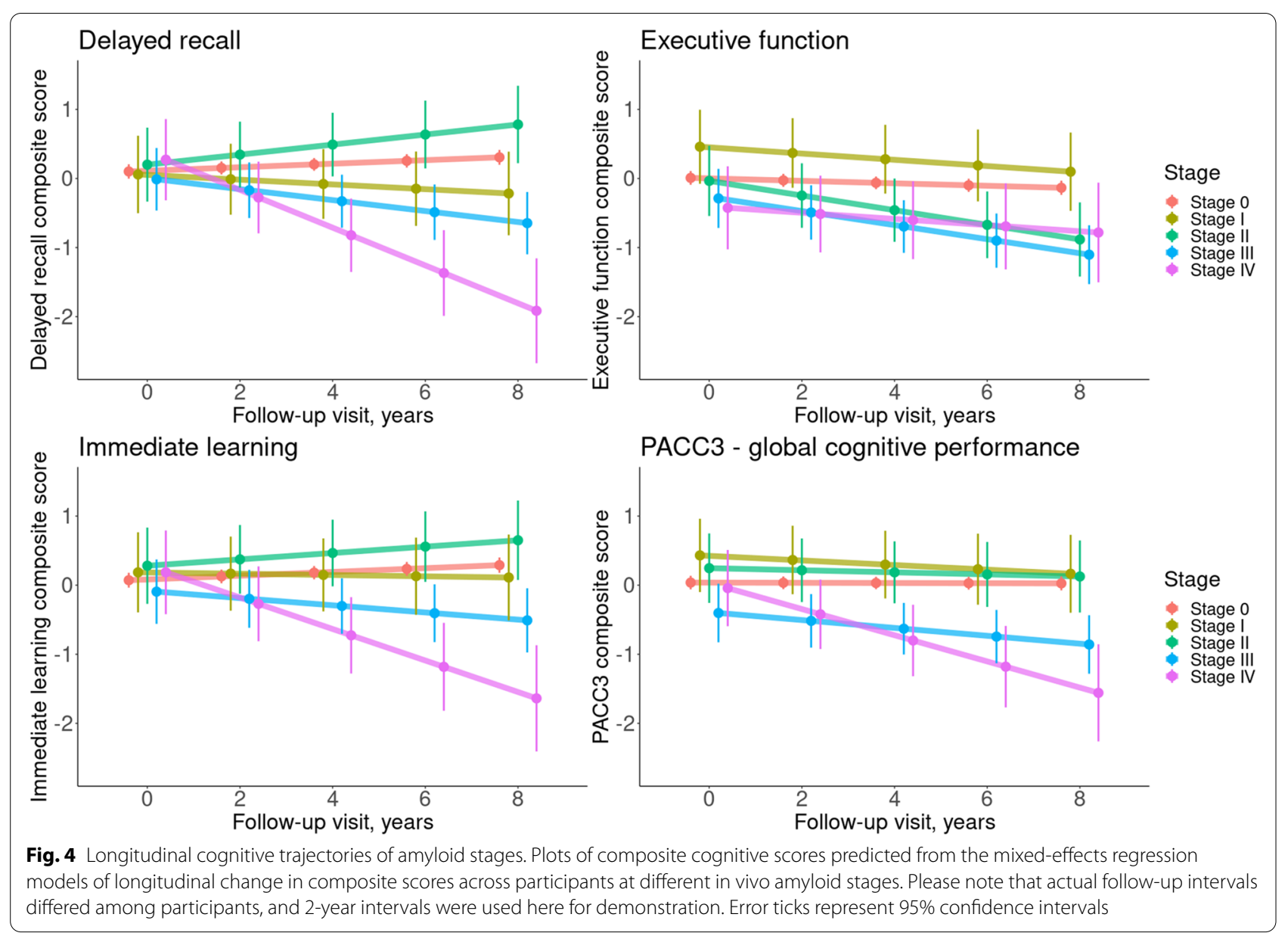

Table 2 Mixed-effects regression models of longitudinal change in composite cognitive scores across baseline amyloid accumulation stages

\begin{tabular}{|c|c|c|c|c|c|c|c|c|}
\hline & \multicolumn{2}{|c|}{$\begin{array}{l}\text { Delayed recall composite } \\
\text { score }\end{array}$} & \multicolumn{2}{|c|}{$\begin{array}{l}\text { Executive function } \\
\text { composite score }\end{array}$} & \multicolumn{2}{|c|}{$\begin{array}{l}\text { Immediate learning } \\
\text { composite score }\end{array}$} & \multicolumn{2}{|c|}{ PACC3 composite score } \\
\hline & Estimate & $t$-statistic & Estimate & t-statistic & Estimate & $t$-statistic & Estimate & $t$-statistic \\
\hline Intercept & -0.338 & -0.542 & $2.829^{* * *}$ & 4.557 & -0.051 & -0.078 & 0.567 & 0.934 \\
\hline Age & $-0.021^{* *}$ & -2.683 & $-0.06^{* * *}$ & -7.765 & $-0.029^{* * *}$ & -3.555 & $-0.04^{* * *}$ & -5.344 \\
\hline Gender & $0.482^{* * *}$ & 5.05 & 0.103 & 1.069 & $0.565^{* * *}$ & 5.655 & $0.528^{* * *}$ & 5.684 \\
\hline Education & $0.054^{* *}$ & 3.214 & $0.039^{*}$ & 2.344 & $0.056^{* *}$ & 3.184 & $0.063^{* * *}$ & 3.826 \\
\hline Follow-up time, years & $0.026^{* * *}$ & 3.889 & $-0.018^{* *}$ & -3.294 & $0.027^{* * *}$ & 4.218 & -0.001 & -0.256 \\
\hline Stage I & -0.043 & -0.149 & 0.451 & 1.624 & 0.114 & 0.378 & 0.393 & 1.43 \\
\hline Stage II & 0.099 & 0.356 & -0.043 & -0.164 & 0.21 & 0.731 & 0.209 & 0.799 \\
\hline Stage III & -0.113 & -0.479 & -0.297 & -1.326 & -0.166 & -0.68 & $-0.44^{*}$ & -1.981 \\
\hline Stage IV & 0.171 & 0.56 & -0.433 & -1.397 & 0.114 & 0.364 & -0.08 & -0.281 \\
\hline Follow-up time $\times$ stage $I$ & -0.06 & -1.593 & -0.027 & -0.897 & -0.037 & -0.99 & -0.032 & -1.007 \\
\hline Follow-up time $\times$ stage $\|$ & 0.047 & 1.227 & $-0.088^{* *}$ & -2.712 & 0.019 & 0.5 & -0.014 & -0.425 \\
\hline Follow-up time $\times$ stage III & $-0.105^{* * *}$ & -3.377 & $-0.084^{* * *}$ & -3.348 & $-0.079^{* *}$ & -2.598 & $-0.056^{*}$ & -2.132 \\
\hline Follow-up time $\times$ stage IV & $-0.299^{* * *}$ & -5.514 & -0.027 & -0.596 & $-0.255^{* * *}$ & -4.783 & $-0.188^{* * *}$ & -4.043 \\
\hline
\end{tabular}

Unstandardized estimates are presented with $t$-statistics. ${ }^{*} p<.05,{ }^{* *} p<.01,{ }^{* * *} p<.001$. For interactions between the follow-up time in years and stage, the stage 0 group acts as a reference. Random intercepts for participants are included to account for multiple measurements 
However, a meta-analysis across 5000 cognitively normal older individuals did not find evidence for a specific effect of amyloid accumulation on memory decline compared to other cognitive domains [48]. Interestingly, participants in stages 0 and II demonstrated a slight improvement in immediate learning and delayed recall over time (Fig. 4, Table 2), which likely reflects practice effects due to repeated exposure to the tests. Such effects have been described in cognitively normal at-risk cohorts before [49], and they are also consistent with previous findings in the WRAP cohort [50]. To compare the predictive value of regional amyloid stages with global PiB-PET signal, we conducted supplementary regression analyses replacing the regional amyloid stages with subgroupings based on a stratification of the range of standard (i.e., non-PVE-corrected) global average DVR values into five equal parts (global DVR groups 0-IV; see Supplementary table 3). Not surprisingly, the global DVR grouping was generally highly correlated with the amyloid stages in the regional staging model (Spearman's rho $=0.78$ ): participants who were assigned into higher DVR groups also tended to be in more advanced regional amyloid stages. However, classification based on global DVR also showed some discrepancies with regional amyloid stages, for example, global DVR group I included participants from all regional amyloid stages.

This underscores the differences between measures of global amyloid load and staging of regional amyloid accumulation. The global DVR stratification was also a significant predictor of longitudinal cognitive decline (Supplementary table 4). Regression analysis with longitudinal cognitive measures suggested that global DVR group III showed a significantly faster decline on all cognitive composite scores. Global DVR group IV showed a significant decline only on delayed recall and immediate learning composite scores, although this observation is limited by the very small number of participants classified into this very advanced global amyloid stage in our study sample.

The faster decline of the global cognitive measure in stages III and IV and a more domain-selective decline in stage II are generally consistent with previous research linking region-specific amyloid deposition to longitudinal cognitive decline in non-demented individuals, although previous studies typically examined considerably older individuals and over shorter follow-up intervals. In our recent ${ }^{18} \mathrm{~F}$-florbetapir PET-based amyloid staging study, we found that higher amyloid stages (from stage II onwards) were associated with a higher risk for progression to mild cognitive impairment in cognitively normal older individuals and subjective memory complainers from two different cohorts [13]. A voxel-based imaging study found that the earliest amyloid-related episodic memory decline among cognitively normal individuals associated with spatially circumscribed increases in regional amyloid-PET signal in the medial and lateral parietal neocortex [51]. Other studies have linked amyloid spread to the striatum and other subcortical regions with a higher risk of cognitive decline among nondemented individuals $[45,52]$. Thus, when subjects were stratified into three stages according to their neocortical and striatal/subcortical radiotracer uptake values, individuals in the most advanced stage with high neocortical and high subcortical signal demonstrated a significantly faster longitudinal decline in cognitive performance compared to both individuals without evidence of amyloid deposition and those with only neocortical amyloid deposition $[45,52]$. Here, we extend these findings to a more comprehensive data-driven regional amyloid staging scheme and a considerably younger at-risk population, thus emphasizing the potential clinical relevance of early detection of regional amyloid deposition even among healthy middle-aged individuals at risk for AD.

\section{Strengths and limitations}

A principal limitation of our study is that although the initial sample size was relatively large, the final number of participants categorized into the different amyloid stages was relatively low, which was also reflected in a low proportion of globally amyloid-positive individuals in the cohort (15\%). Previous studies on the WRAP cohort have reported relatively higher rates of amyloid positivity (approximately $20 \%$ depending on the assessed subcohort and study visit), which could be due to the use of different cortical masks for calculating the global average signal $[53,54]$. The regional staging approach employed in our study uses several methodological settings that aim to increase the correspondence of regional PiB-PET measurements with actual amyloid accumulation, including the use of DVR images [55], PVE correction [28, 56], and region-specific amyloid positivity thresholds [17]. In complementary analyses, we assessed the potential contributions of these methodological aspects by estimating additional amyloid staging models using alternative PET processing methods and threshold definitions (Supplementary figures 1-3; Supplementary tables 1, 5, and 6). Generally, the staging models estimated using SUVR images and non-PVE-corrected data showed only relatively minor differences compared to the main model with PVE-corrected DVR data (Supplementary figure 3), such that the respective regional amyloid positivity frequencies were highly correlated (rho $\sim 0.86$; Supplementary table 5). By contrast, the use of a constant universal cutoff for all brain regions had a major influence on the regional staging model, yielding low-rank correlations with the regional amyloid positivity frequencies of all 
other models (rho $\leq 0.22$ ). While the highest frequencies among cortical regions were similarly observed in anterior and posterior midline regions, regional frequencies were also very high in subcortical structures, including the striatum and thalamus (54\% and $95 \%$, respectively), when using a constant cutoff. These high frequencies of amyloid positivity could likely be attributed to the high influence of universal, non-specific white matter binding in these subcortical regions embedded within the brain's white matter. All methods used for estimating region-specific cutoffs (2D-GMM, 1D-GMM, young subsample; see Supplementary figure 1) suggested considerably higher cutoffs for these structures compared to the thresholds estimated for most cortical regions. In terms of individual staging results, all models based on alternative PET processing strategies resulted in one or two additional participants being classified as non-stageable, indicating a minor advantage of the main model (using PVE-corrected DVR maps and region-specific thresholds) in this regard.

Our employed regional amyloid staging approach also has some limitations. First, the staging model in the present study was estimated using data from a single radiotracer, which could limit its applicability to data obtained with other tracers as compared to staging models estimated across multi-tracer amyloid-PET data [57]. Second, the current staging approach assumes a single trajectory for regional amyloid accumulation across all individuals. Although the very low number of nonstageable participants argues against major deviations of regional amyloid deposition across the four larger anatomical divisions estimated by the staging model, individual variance may still be present at a more granular spatial resolution not considered by the model (e.g., variable involvement of inferior and lateral temporal, anterior medial, and posterior medial cortical regions, all represented within the same stage I in the model). Moreover, other pathologies that often co-occur with AD-typical amyloid accumulation, such as cerebral amyloid angiopathy (CAA), may contribute to individual deviations from the estimated staging model. In this context, it is interesting to note that three out of the four non-stageable participants demonstrated amyloid positivity in the occipital pole, which showed an overall low frequency of amyloid positivity in this sample but had previously been associated with CAA [58].

\section{Conclusion}

In summary, in the current study, we applied the in vivo amyloid staging approach that was developed and replicated previously using ${ }^{18} \mathrm{~F}$-florbetapir PET data to ${ }^{11} \mathrm{C}$-PiB-PET data from a relatively young cohort enriched with risk for AD. The cross-sectionally estimated staging model utilizing region-specific thresholds was largely consistent with previous models established based on ${ }^{18} \mathrm{~F}$-florbetapir PET and was further supported by model-conform longitudinal stage transitions as well as by the pattern of longitudinal emergence of regional amyloid positivity in participants who had no amyloid-positive regions at baseline. Using exceptionally long clinical follow-up data we could demonstrate amyloid stage-proportional risks of cognitive decline even in this comparably young at-risk cohort. Together, these data support the robustness and clinical utility of in vivo amyloid staging for risk stratification of the preclinical phase of AD.

\section{Abbreviations \\ AD: Alzheimer's disease; APOE: Apolipoprotein E; Aß: Beta-amyloid; DVR: Distribution volume ratio; GMM: Gaussian mixture model; MCl: Mild cognitive impairment; MMSE: Mini-Mental State Examination; MRI: Magnetic resonance imaging; PACC3: Three test version of the preclinical Alzheimer's cogni- tive composite; PET: Positron emission tomography; ${ }^{11} \mathrm{C}-\mathrm{PiB}:{ }^{11} \mathrm{C}$ Pittsburgh Compound B; THEO-DEL-REC: Delayed recall composite score; THEO-EXEC-FN: Executive function composite score; THEO-IMM-LRN: Immediate learning composite score; WRAP: Wisconsin Registry for Alzheimer's Prevention.}

\section{Supplementary Information}

The online version contains supplementary material available at https://doi. org/10.1186/s13195-021-00918-0.

Additional file 1: Effect of different methods for estimating regional amyloid positivity thresholds on staging models of regional amyloid progression. Figure S1. Regional amyloid-positivity frequencies across three methods used for estimating amyloid accumulation thresholds. Table S1. Correlations between amyloid-positivity frequencies based on different amyloid-positivity thresholds. Table 2. Sample characteristics by longitudinal amyloid progression status. Table 3. Correspondence between regional amyloid staging results and subgroups stratified by global DVR signal. Table 4. Mixed-effects regression models of longitudinal change in composite cognitive scores across groups of participants stratified by global non-PVE-corrected ${ }^{11} \mathrm{C}$-PiB-PET DVR signal. Effect of different PET processing methods on estimated staging models of regional amyloid progression Figure S2. Correspondence between global DVR signal extracted from non-PVE-corrected and PVE-corrected ${ }^{11} \mathrm{C}$-PiB-PET data. Figure S3. Regional amyloid-positivity frequencies across different PET processing methods. Table S5. Correlations between amyloid-positivity frequencies based on different PET processing methods. Table S6. Summary of individual staging results across models with different PET processing methods.

\section{Acknowledgements}

Not applicable.

\section{Authors' contributions}

TJB, OO, and SCJ contributed to the data acquisition. TJB contributed to the image processing, quantification, and quality control; IJ, SJT, and MJG designed and conceptualized the study. FL, IJ, SJT, and MJG analyzed the data and drafted the manuscript. All authors contributed to the review and editing of the manuscript and approved the final version.

Funding

The present study was funded in part by a grant from the Alzheimer Forschung Initiative e.V. to MJG (grant number \#16037). Michel J. Grothe is supported by the "Miguel Servet" program [CP19/00031] and a research grant 
[PI20/00613] of the Instituto de Salud Carlos III-Fondo Europeo de Desarrollo Regional (ISCIII-FEDER). Additional funding for the WRAP study and PET imaging was provided by NIH AG027161 and AG021155, and the Alzheimer's Association AARF-19-614533. Open Access funding enabled and organized by Projekt DEAL.

\section{Availability of data and materials}

The data analyzed in this study were acquired from the Wisconsin Registry for Alzheimer's Prevention (WRAP) study. Data are available for authorized researchers upon request subject to a decision by the WRAP Science Executive Committee. For data requests, please refer to https://wrap.wisc.edu/datarequests/.

\section{Declarations}

\section{Ethics approval and consent to participate}

Study procedures within WRAP were approved by the Institutional Review Board of the University of Wisconsin. Each participant provided informed consent before participating in the study.

\section{Consent for publication}

Not applicable.

\section{Competing interests}

$\mathrm{SCJ}$ is a scientific advisor to Roche Diagnostics and received grant funding from Cerveau Technologies. SJT participated in scientific advisory boards of Roche Pharma AG and MSD and received lecture fees from Roche, MSD, and Biogen. FL, IJ, TJB, OO, and MJG declare that they have no competing interests.

\section{Author details}

'German Center for Neurodegenerative Diseases (DZNE), Rostock/Greifswald, Rostock, Germany. ${ }^{2}$ Division of Geriatrics and Gerontology, Department of Medicine, University of Wisconsin-Madison School of Medicine and Public Health, Madison, WI, USA. ${ }^{3}$ Wisconsin Alzheimer's Disease Research Center, University of Wisconsin-Madison School of Medicine and Public Health, Madison, WI, USA. ${ }^{4}$ Wisconsin Alzheimer's Institute, University of WisconsinMadison School of Medicine and Public Health, Madison, WI, USA. ${ }^{5}$ Geriatric Research Education and Clinical Center, William S. Middleton Memorial Veterans Hospital, Madison, WI, USA. ${ }^{6}$ Department of Psychosomatic Medicine, University of Rostock, Rostock, Germany. ${ }^{7}$ Unidad de Trastornos del Movimiento, Servicio de Neurología y Neurofisiología Clínica, Instituto de Biomedicina de Sevilla, Hospital Universitario Virgen del Rocío/CSIC/Universidad de Sevilla, s/n, 41013 Seville, Spain.

Received: 18 May 2021 Accepted: 11 October 2021

Published online: 21 October 2021

\section{References}

1. Braak H, Thal DR, Ghebremedhin E, Del Tredici K. Stages of the pathologic process in Alzheimer disease: age categories from 1 to 100 years. J Neuropathol Exp Neurol. 2011;70(11):960-9.

2. Jack CR Jr, Knopman DS, Jagust WJ, Petersen RC, Weiner MW, Aisen PS, et al. Tracking pathophysiological processes in Alzheimer's disease: an updated hypothetical model of dynamic biomarkers. Lancet Neurol. 2013;12(2):207-16.

3. Sperling R, Mormino E, Johnson K. The evolution of preclinical Alzheimer's disease: implications for prevention trials. Neuron. 2014;84(3):608-22.

4. Thal DR, Rub U, Orantes M, Braak H. Phases of A beta-deposition in the human brain and its relevance for the development of AD. Neurology. 2002;58(12):1791-800.

5. Clark CM, Pontecorvo MJ, Beach TG, Bedell BJ, Coleman RE, Doraiswamy PM, et al. Cerebral PET with florbetapir compared with neuropathology at autopsy for detection of neuritic amyloid- $\beta$ plaques: a prospective cohort study. The Lancet Neurology. 2012;1 1 (8):669-78.

6. Sabri O, Seibyl J, Rowe C, Barthel H. Beta-amyloid imaging with florbetaben. Clin Transl Imaging. 2015;3(1):13-26.
7. Villemagne VL, Ong K, Mulligan RS, Holl G, Pejoska S, Jones G, et al. Amyloid imaging with ${ }^{18} \mathrm{~F}$-florbetaben in Alzheimer disease and other dementias. J Nucl Med. 2011;52(8):1210-7.

8. Villeneuve S, Rabinovici GD, Cohn-Sheehy BI, Madison C, Ayakta N, Ghosh PM, et al. Existing Pittsburgh Compound-B positron emission tomography thresholds are too high: statistical and pathological evaluation. Brain. 2015;138(Pt 7):2020-33.

9. Braak H, Braak E. Neuropathological stageing of Alzheimer-related changes. Acta Neuropathol. 1991;82(4):239-59.

10. Grothe MJ, Barthel H, Sepulcre J, Dyrba M, Sabri O, Teipel SJ, et al. In vivo staging of regional amyloid deposition. Neurology. 2017;89(20):2031-8.

11. Sakr FA, Grothe MJ, Cavedo E, Jelistratova I, Habert MO, Dyrba M, et al. Applicability of in vivo staging of regional amyloid burden in a cognitively normal cohort with subjective memory complaints: the INSIGHTpreAD study. Alzheimers Res Ther. 2019;1 1(1):15.

12. Teipel SJ, Temp AGM, Levin F, Dyrba M, Grothe MJ, Alzheimer's Disease Neuroimaging I. Association of PET-based stages of amyloid deposition with neuropathological markers of Abeta pathology. Ann Clin Transl Neurol. 2021;8(1):29-42.

13. Teipel SJ, Dyrba M, Chiesa PA, Sakr F, Jelistratova I, Lista S, et al. In vivo staging of regional amyloid deposition predicts functional conversion in the preclinical and prodromal phases of Alzheimer's disease. Neurobiol Aging. 2020;93:98-108.

14. Schmidt ME, Chiao P, Klein G, Matthews D, Thurfiell L, Cole PE, et al. The influence of biological and technical factors on quantitative analysis of amyloid PET: points to consider and recommendations for controlling variability in longitudinal data. Alzheimers Dement. 2015;1 1(9):1050-68.

15. Chetelat G, Murray ME. Amyloid PET scan: staging beyond reading? Neurology. 2017;89(20):2029-30.

16. Joshi AD, Pontecorvo MJ, Clark CM, Carpenter AP, Jennings DL, Sadowsky $\mathrm{CH}$, et al. Performance characteristics of amyloid PET with florbetapir $\mathrm{F}$ 18 in patients with Alzheimer's disease and cognitively normal subjects. J Nucl Med. 2012;53(3):378-84.

17. Jelistratova I, Teipel SJ, Grothe MJ. Longitudinal validity of PETbased staging of regional amyloid deposition. Hum Brain Mapp. 2020;41(15):4219-31.

18. Landau SM, Thomas BA, Thurfell L, Schmidt M, Margolin R, Mintun M, et al. Amyloid PET imaging in Alzheimer's disease: a comparison of three radiotracers. Eur J Nucl Med Mol Imaging. 2014;41 (7):1398-407.

19. Beach TG, Maarouf CL, Intorcia A, Sue LI, Serrano GE, Lu M, et al. Antemortem-postmortem correlation of florbetapir $\left({ }^{18} \mathrm{~F}\right)$ PET amyloid imaging with quantitative biochemical measures of $A \beta_{42}$ but not $A \beta_{40}$.J Alzheimers Dis. 2018;61(4):1509-16.

20. Ni R, Gillberg PG, Bergfors A, Marutle A, Nordberg A. Amyloid tracers detect multiple binding sites in Alzheimer's disease brain tissue. Brain. 2013;136(Pt 7):2217-27.

21. Seo SW, Ayakta N, Grinberg LT, Villeneuve S, Lehmann M, Reed B, et al. Regional correlations between $\left[{ }^{11} \mathrm{C}\right] \mathrm{PIB}$ PET and post-mortem burden of amyloid-beta pathology in a diverse neuropathological cohort. Neuroimage Clin. 2017;13:130-7.

22. Ikonomovic MD, Buckley CJ, Abrahamson EE, Kofler JK, Mathis CA, Klunk WE, et al. Post-mortem analyses of PiB and flutemetamol in diffuse and cored amyloid-beta plaques in Alzheimer's disease. Acta Neuropathol. 2020;140(4):463-76.

23. Johnson SC, Christian BT, Okonkwo OC, Oh JM, Harding S, Xu G, et al. Amyloid burden and neural function in people at risk for Alzheimer's disease. Neurobiol Aging. 2014;35(3):576-84.

24. Logan J, Fowler JS, Volkow ND, Wang GJ, Ding YS, Alexoff DL. Distribution volume ratios without blood sampling from graphical analysis of PET data. J Cereb Blood Flow Metab. 1996;16(5):834-40.

25. Lopresti BJ, Klunk WE, Mathis CA, Hoge JA, Ziolko SK, Lu X, et al. Simplified quantification of Pittsburgh Compound B amyloid imaging PET studies: a comparative analysis. J Nucl Med. 2005;46(12):1959-72.

26. Grothe $M$, Heinsen $H$, Teipel $S$. Longitudinal measures of cholinergic forebrain atrophy in the transition from healthy aging to Alzheimer's disease. Neurobiol Aging. 2013;34(4):1210-20.

27. Ashburner J. A fast diffeomorphic image registration algorithm. Neuroimage. 2007;38(1):95-113.

28. Gonzalez-Escamilla G, Lange C, Teipel S, Buchert R, Grothe MJ. Alzheimer's Disease Neuroimaging I. PETPVE12: an SPM toolbox for partial volume 
effects correction in brain PET - application to amyloid imaging with AV45-PET. Neuroimage. 2017;147:669-77.

29. Muller-Gartner HW, Links JM, Prince JL, Bryan RN, McVeigh E, Leal JP, et al. Measurement of radiotracer concentration in brain gray matter using positron emission tomography: MRI-based correction for partial volume effects. J Cereb Blood Flow Metab. 1992;12(4):571-83.

30. Teipel S, Heinsen H, Amaro E Jr, Grinberg LT, Krause B, Grothe M, et al. Cholinergic basal forebrain atrophy predicts amyloid burden in Alzheimer's disease. Neurobiol Aging. 2014;35(3):482-91.

31. Mormino EC, Betensky RA, Hedden T, Schultz AP, Ward A, Huijbers W, et al. Amyloid and APOE $\varepsilon 4$ interact to influence short-term decline in preclinical Alzheimer disease. Neurology. 2014;82(20):1760-7.

32. Hanseeuw BJ, Betensky RA, Schultz AP, Papp KV, Mormino EC, Sepulcre $J$, et al. Fluorodeoxyglucose metabolism associated with tau-amyloid interaction predicts memory decline. Ann Neurol. 2017;81(4):583-96.

33. Jack CR Jr, Bennett DA, Blennow K, Carrillo MC, Feldman HH, Frisoni GB, et al. A/T/N: an unbiased descriptive classification scheme for Alzheimer disease biomarkers. Neurology. 2016;87(5):539-47.

34. Josephs KA, Murray ME, Whitwell JL, Tosakulwong N, Weigand SD, Petrucelli $\mathrm{L}$, et al. Updated TDP-43 in Alzheimer's disease staging scheme. Acta neuropathologica. 2016;131(4):571-85.

35. Jonaitis EM, Koscik RL, Clark LR, Ma Y, Betthauser TJ, Berman SE, et al. Measuring longitudinal cognition: individual tests versus composites. Alzheimers Dement (Amst). 2019;11:74-84.

36. Levin F, Ferreira D, Lange C, Dyrba M, Westman E, Buchert R, et al. Datadriven FDG-PET subtypes of Alzheimer's disease-related neurodegeneration. Alzheimers Res Ther. 2021;13(1):49.

37. Cho H, Choi JY, Hwang MS, Kim YJ, Lee HM, Lee HS, et al. In vivo cortical spreading pattern of tau and amyloid in the Alzheimer disease spectrum. Ann Neurol. 2016;80(2):247-58.

38. Rodrigue KM, Kennedy KM, Devous MD Sr, Rieck JR, Hebrank AC, DiazArrastia $\mathrm{R}$, et al. $\beta$-Amyloid burden in healthy aging: regional distribution and cognitive consequences. Neurology. 2012;78(6):387-95.

39. Gonneaud J, Arenaza-Urquijo EM, Mezenge F, Landeau B, Gaubert M, Bejanin A, et al. Increased florbetapir binding in the temporal neocortex from age 20 to 60 years. Neurology. 2017;89(24):2438-46.

40. Mattsson N, Palmqvist S, Stomrud E, Vogel J, Hansson O. Staging $\beta$-amyloid pathology with amyloid positron emission tomography. JAMA Neurol. 2019;76(11):1319-29.

41. Palmqvist S, Scholl M, Strandberg O, Mattsson N, Stomrud E, Zetterberg $\mathrm{H}$, et al. Earliest accumulation of beta-amyloid occurs within the defaultmode network and concurrently affects brain connectivity. Nat Commun. 2017:8(1):1214

42. Klunk WE, Price JC, Mathis CA, Tsopelas ND, Lopresti BJ, Ziolko SK, et al. Amyloid deposition begins in the striatum of presenilin-1 mutation carriers from two unrelated pedigrees. J Neurosci. 2007;27(23):6174-84.

43. Villemagne VL, Ataka S, Mizuno T, Brooks WS, Wada Y, Kondo M, et al. High striatal amyloid beta-peptide deposition across different autosomal Alzheimer disease mutation types. Arch Neurol. 2009;66(12):1537-44.

44. Koivunen J, Verkkoniemi A, Aalto S, Paetau A, Ahonen JP, Viitanen M, et al. PET amyloid ligand [11C]PIB uptake shows predominantly striatal increase in variant Alzheimer's disease. Brain. 2008;131(Pt 7):1845-53.
45. Hanseeuw BJ, Betensky RA, Mormino EC, Schultz AP, Sepulcre J, Becker $J A$, et al. PET staging of amyloidosis using striatum. Alzheimers Dement. 2018;14(10):1281-92.

46. Ferreira D, Machado A, Molina Y, Nieto A, Correia R, Westman E, et al. Cognitive variability during middle-age: possible association with neurodegeneration and cognitive reserve. Front Aging Neurosci. 2017;9:188.

47. Farrell ME, Kennedy KM, Rodrigue KM, Wig G, Bischof GN, Rieck JR, et al. Association of longitudinal cognitive decline with amyloid burden in middle-aged and older adults: evidence for a dose-response relationship. JAMA Neurol. 2017;74(7):830-8.

48. Baker JE, Lim YY, Pietrzak RH, Hassenstab J, Snyder PJ, Masters CL, et al. Cognitive impairment and decline in cognitively normal older adults with high amyloid-beta: a meta-analysis. Alzheimers Dement (Amst). 2017:6:108-21.

49. Teipel SJ, Cavedo E, Lista S, Habert MO, Potier MC, Grothe MJ, et al. Effect of Alzheimer's disease risk and protective factors on cognitive trajectories in subjective memory complainers: an INSIGHT-preAD study. Alzheimers Dement. 2018;14(9):1126-36.

50. Clark LR, Berman SE, Norton D, Koscik RL, Jonaitis E, Blennow K, et al. Age-accelerated cognitive decline in asymptomatic adults with CSF betaamyloid. Neurology. 2018;90(15):e1306-e15.

51. Farrell ME, Chen X, Rundle MM, Chan MY, Wig GS, Park DC. Regional amyloid accumulation and cognitive decline in initially amyloid-negative adults. Neurology. 2018;91(19):e1809-e21.

52. Cho SH, Shin JH, Jang H, Park S, Kim HJ, Kim SE, et al. Amyloid involvement in subcortical regions predicts cognitive decline. Eur J Nucl Med Mol Imaging. 2018;45(13):2368-76.

53. Sprecher KE, Bendlin BB, Racine AM, Okonkwo OC, Christian BT, Koscik RL, et al. Amyloid burden is associated with self-reported sleep in nondemented late middle-aged adults. Neurobiol Aging. 2015;36(9):2568-76.

54. Koscik RL, Betthauser TJ, Jonaitis EM, Allison SL, Clark LR, Hermann BP, et al. Amyloid duration is associated with preclinical cognitive decline and tau PET. Alzheimers Dement (Amst). 2020;12(1):e12007.

55. Lopes Alves I, Heeman F, Collij LE, Salvado G, Tolboom N, Vilor-Tejedor $\mathrm{N}$, et al. Strategies to reduce sample sizes in Alzheimer's disease primary and secondary prevention trials using longitudinal amyloid PET imaging. Alzheimers Res Ther. 2021;13(1):82.

56. Rullmann M, Dukart J, Hoffmann KT, Luthardt J, Tiepolt S, Patt M, et al. Partial-volume effect correction improves quantitative analysis of 18F-florbetaben beta-amyloid PET scans. J Nucl Med. 2016;57(2):198-203.

57. Collij LE, Heeman F, Salvado G, Ingala S, Altomare D, de Wilde A, et al. Multitracer model for staging cortical amyloid deposition using PET imaging. Neurology. 2020;95(11):e1538-e53.

58. Charidimou A, Farid K, Tsai HH, Tsai LK, Yen RF, Baron JC. Amyloid-PET burden and regional distribution in cerebral amyloid angiopathy: a systematic review and meta-analysis of biomarker performance. J Neurol Neurosurg Psychiatry. 2018;89(4):410-7.

\section{Publisher's Note}

Springer Nature remains neutral with regard to jurisdictional claims in published maps and institutional affiliations.

\footnotetext{
Ready to submit your research? Choose BMC and benefit from:

- fast, convenient online submission

- thorough peer review by experienced researchers in your field

- rapid publication on acceptance

- support for research data, including large and complex data types

- gold Open Access which fosters wider collaboration and increased citations

- maximum visibility for your research: over $100 \mathrm{M}$ website views per year
}

At BMC, research is always in progress.

Learn more biomedcentral.com/submissions 